Nervenarzt 2010 $81: 1427-1428$

DOI 10.1007/s00115-010-3102-9

Online publiziert: 27. November 2010

๑) Springer-Verlag 2010

\author{
C. Weiller ${ }^{1} \cdot$ T. Brandt $^{2}$ \\ ${ }^{1}$ Neurologische Universitätsklinik Freiburg, Freiburg i.Br. \\ ${ }^{2}$ Integriertes Forschungs- und Behandlungszentrum für Schwindel-, \\ Gleichgewichts- und Augenbewegungsstörungen (IFB), \\ Neurologische Klinik der LMU München, München
}

\title{
Funktionelle Bildgebung in der Neurologie
}

Warum ein neurologisches Themenheft zur funktionellen Bildgebung in Der Nervenarzt? Während früher nur aus Autopsie oder später aus Läsionsstudien mit Computer- (CT) oder heute mit Magnetresonanztomographie (MRT) auf die Funktion des Gehirns geschlossen wurde [8], begann mit der funktionellen Bildgebung eine neue Ära. Wurden in den ersten 10 Jahren aufgabenbezogene „Aktivierungen" räumlich zugeordnet, heißt das Ziel der funktionellen Bildgebung heute, auch die Verbindungen dazwischen, also ganze „Netzwerke“ zu beschreiben. Eine Analogie ist die Reise in einem Flugzeug, in dem man nachts von oben nur die (beleuchteten) Städte sieht, tagsüber aber auch die Autobahnen dazwischen oder sogar die Dichte des Verkehrs zu einer bestimmten Uhrzeit.

Es ist ein Fortschritt, neben den Städten oder Aktivierungen auch die Autobahnen oder Straßen zu kennen, aber das eigentliche Problem ist ein anderes: Funktion sitzt weder in Arealen, noch wird sie durch die Verbindung von Arealen definiert, sondern sie entsteht durch Interaktion. Hier liegt ein klassischer Fehler der Neurologie, die Annahme der Unabhängigkeit von Zentren, exemplarisch dargestellt an einem sog. Diskonnektionssyndrom, der Leitungsaphasie. Hierbei soll es durch Läsion des Fasciculus arcuatus, der die Sprachzentren im Temporal- („Wernicke") und Frontallappen („Broca“) verbindet, zu einer isolierten Störung des Nachsprechens bei erhaltenem Wortverständnis und erhaltener Spontansprache kommen [1]. Die Annahme dabei ist, dass der Temporallappen allein ausreiche für
Sprachverständnis und der untere Frontallappen allein für die spontane Sprachproduktion, d. h. dass beide unabhängig voneinander funktionieren. Dem ist nicht so. Semantische Aufgaben aktivieren Broca-Areale und Spontansprache den Temporallappen $[5,11,15]$. Läsionen des Temporallappens können eine WernickeAphasie hervorrufen, aber diese entsteht auch bei Läsionen außerhalb des Temporallappens [20].

\section{$>$ Funktion entsteht durch kontextabhängige Interaktion}

Wir gehen heute davon aus, dass die meisten Funktionen des Gehirns in weit verzweigten, überlappenden Systemen organisiert sind [12], übrigens eine Annahme, die sich schon bei Wernicke findet $[18,19]$. Eine Aktivierung in einem Areal oder die Benutzung einer bestimmten Verbindung kann je nach Zusammenhang völlig unterschiedliche Funktionen repräsentieren, d. h. es kommt auf den Kontext an [3, 14]. Funktion entsteht durch kontextabhängige Interaktion. Funktion und Domäne werden durch die jeweils zeitbezogen verbundenen Gebiete definiert. Das bedeutet nicht, dass eine Verbindung nicht für eine bestimmte Funktion notwendig ist, wie z. B. der Fasciculus arcuatus für das Nachsprechen. Allerdings führt eine Läsion dieser Verbindung nicht notwendigerweise zu einer isolierten Störung dieser Funktion, sondern zu einer ganz neuen phänomenologischen Konstellation.

Schon die ersten Studien bei Schlaganfallpatienten zeigten, dass die Funktionserholung mit einer Reorganisation der verbleibenden Anteile des Netzes und soweit möglich einer Normalisierung einhergeht $[16,17]$. Daraus ergibt sich die Notwendigkeit der Bestimmung der jeweiligen Netzwerkarchitektur und der Wirkweise von Therapien auf der systemischen Ebene [13].

Das Erkennen solcher systemischer Netze, bestehend aus Knotenpunkten (den fMRT-Aktivierungen) und deren Verbindungen bzw. funktionellen Interaktionen, ist durch Kombinationen neuester Verfahren zur Bildgebung beim Menschen möglich geworden. Aus den Zeitreihen der fMRT-Untersuchungen lassen sich Korrelationen oder modellgestützte Interaktionen bestimmen (Konnektivität) $[2,4][10]$. Der anatomische Verlauf der Verbindungsbahnen zwischen den Knotenpunkten lässt sich mit der Diffusionstensorbildgebung abschätzen. Moleküle diffundieren leichter entlang als quer zu den Axonen, die in den Trakten gebündelt sind. Diese Diffusionsbewegung kann näherungsweise durch einen Diffusionstensor beschrieben werden, welcher sich mit diffusionsgewichteter Bildgebung messen lässt. Probabilistische oder deterministische Tracking-Algorithmen zeigen den wahrscheinlichsten Verlauf der Trakte an. Damit lässt sich insbesondere der Verlauf von Verbindungen zwischen je zwei durch fMRT-Aktivierungen definierten Knotenpunkten nachvollziehen. Aus allen möglichen Verbindungen lassen sich dann die für die aktuelle Funktion wichtigen auswählen. Erste Ansätze der Beschreibung derartige kompletter Netzwerke für Sprache und visuell räumliche Aufmerksamkeit finden sich in den 
Betrachtungen zur Aphasie (Saur, in diesem Heft) und Neglekt (Umarova, in diesem Heft). Im Beitrag „Apraxie“ (Weiss, in diesem Heft) entschlüsselt die funktionelle Bildgebung unterschiedliche Areale innerhalb des motorischen Netzwerkes, die für Einzelstörungen der höheren motorischen Kognition, wie Imitation von Gesten (Dysfunktion des linken inferioren parietalen Kortex), der Pantomime (Dysfunktion im linken inferioren frontalen Kortex), oder Defizite des Objektgebrauchs bei Dysfunktion des frontoparietalen Netzwerkes verantwortlich sind.

In einem Magnetresonanztomographen kann man nicht laufen, um so wichtiger ist der Nachweis, dass identische kortikale und subkortikale Lokomotionszentren, wie sie sich bei realer Lokomotion im PET darstellen, denen der vorgestellten, imaginierten, Lokomotion im fMRI entsprechen [9]. Dies ermöglicht reliable Gruppenvergleiche der unterschiedlichen Lokomotionsstörungen im fMRI bei neurodegenerativen Erkrankungen wie Parkinson oder progressiver supranukleärer Blicklähmung (Jahn und Zwergal, in diesem Heft). Hieraus lassen sich neue Therapiestrategien in Form inhibitorischer oder exitatorischer elektrischer Reizung im präfrontalen Kortex, präzentralen Gyrus, subthalamischen Nukleus oder pedunkulopontinen/kuneiformen Nukleus entwickeln.

Die kritische Anwendung mathematischer Methoden auf neurowissenschaftliche Daten hilft, das nichtparametrische Verhalten des Gehirns besser zu verstehen. So gelang es, mit den sog. „support vector machines" sowohl eine objektive Diagnose aus MRT-Bildern für den M. Alzheimer zu generieren (Klöppel, in diesem Heft) als auch aus dem Muster der Aktivierung auf die Prognose bei Aphasie zu schließen (Saur, in diesem Heft). Der Einsatz künstlicher neuronaler Netzwerke, die nach einem Training treffsicher Musterklassifikationen vornehmen, wird eine zunehmende diagnostische Bedeutung gewinnen.

\section{- Welche Konsequenzen können sich ergeben?}

Wenn es gelingt, den systemischen Mechanismus von Therapien (z. B. „cons- traint induced movement therapy“ (CIMT) $[6,7]-$ d. h. das Netz, welches eine Therapie benutzt - zu charakterisieren, wird eine Zuordnung von Patienten zu geeigneten Therapien möglich; es werden langfristig nur die profitieren, bei denen wesentliche Teile des für die Therapie erforderlichen Netzes erhalten sind. Verstehen wir die Krankheitsprozesse auf systemischer Ebene, sind Diagnosen genauer oder früher möglich. Bisher war es schwierig, den Effekt von Pharmaka besonders auf höhere kognitive Leistungen abzubilden, jetzt kann der Einfluss auf die Interaktion im Netz untersucht werden. Schließlich helfen uns Krankheiten, das Gehirn besser zu verstehen. Die kleinste, praktisch verwendbare Funktionseinheit im Gehirn mag durch deren Alteration bestimmt sein. Wir wissen nicht, in welchen basalen Funktionseinheiten das Gehirn „denkt“. Bestimmt nicht in großen Kategorien wie Satzverständnis und auch nicht entsprechend unserer normalverteilten Tests oder in einfachen Subtraktionen. Das sorgfältige Studium der begrenzten Zahl pathologischer Abweichungen war schon immer die Domäne der Neurologie, zugegebenermaßen haben sich die technischen, experimentellen und mathematischen Methoden deutlich verbessert. Die funktionelle Bildgebung hat in der Neurologie längst eine Renaissance der topographischen Anatomie, der Beziehung von Struktur und Funktion, eingeleitet.

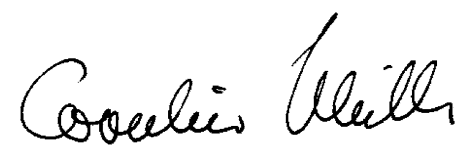

Prof. Dr. C. Weiller

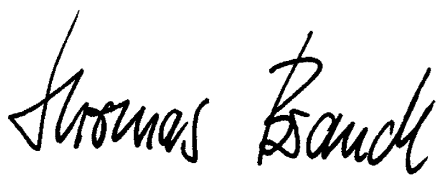

Prof. Dr. T. Brandt

\section{Korrespondenzadresse}

Prof. Dr. C. Weiller

Neurologische Universitätsklinik Freiburg Breisacher Straße 64, 79106 Freiburg i.Br. cornelius.weiller@uniklinik-freiburg.de

\section{Literatur}

1. Benson DF, Sheramata W, Bouchard R et al (1973) Conduction aphasia. Arch Neurol 28:339-346

2. Büchel C, Coull JT, Friston KJ (1999) The predictive value of changes in effective connectivity for human learning. Science 283:1538-1541

3. Cohen J, Stewart I (1995) The collapse of chaos: discovering simplicity in a complex world. Pinguin Science, London

4. Friston KJ, Buechel C, Fink GR et al (1997) Psychophysiological and modulatory interactions in neuroimaging. Neuroimage 6:218-229

5. Grodzinsky Y, Santi A (2008) The battle for Broca's region. Trends Cog Sci 12:474-480

6. Hamzei F, Dettmers C, Rijntjes M, Weiller C (2008) The effect of cortico-spinal tract damage on primary sensorimotor cortex activation after rehabilitation therapy. Exp Brain Res 190:329-336

7. Hamzei F, Liepert J, Dettmers C et al (2006) Two different reorganization patterns after rehabilitative therapy: an exploratory study with $\mathrm{FMRI}$ and TMS. Neuroimage 31:710-720

8. Karnath HO, Fruhmann Berger M et al (2004) The anatomy of spatial neglect based on voxelwise statistical analysis: a study of 140 patients. Cereb Cortex 14:1164-1172

9. la Fougere C, Zwergal A, Rominger A et al (2010) Real versus imagined locomotion: a [18F]-FDG PET-fMRI comparison. Neuroimage 50:1589-1598

10. Mader W, Feess D, Lange R et al. (2008) On the detection of direct directed information flow in fMRI. IEEE J Sel Topics Signal Process 2:965-974

11. Matsumoto R, Nair DR, LaPresto E et al (2004) Functional connectivity in the human language system: a cortico-cortical evoked potential study. Brain 127:2316-2330

12. Mesulam M (1990) Large-scale neurocognitive networks and distributed processing for attention, language, memory. Ann Neurol 28:597-613

13. Rijntjes M (2006) Mechanisms of recovery in stroke patients with hemiparesis or aphasia: new insights, old questions and the meaning of therapies. Curr Opin Neurol 19:76-83

14. Vadia E, Haalman I, Abeles M et al (1995) Dynamics of neuronal interactions in monkey cortex in relation to behavioural events. Nature 373:515-518

15. Vigneau M, Beaucousin V, Herve PY et al (2006) Meta-analyzing left hemisphere language areas: phonology, semantics, and sentence processing. Neuroimage 30:1414-1432

16. Weiller C, Chollet F, Friston KJ et al (1992) Functional reorganization of the brain in recovery from striatocapsular infarction in man. Ann Neurol 31:463-472

17. Weiller C, Rijntjes M (1999) Learning, plasticity and recovery in the central nervous system. Exp Brain Res 128:134-138

18. Wernicke C (1906) Der aphasische Symptomenkomplex. In: Leyden E (ed) Deutsche Klinik am Eingang des zwanzigsten Jahrhunderts in akademischen Vorlesungen. Urban \& Schwarzenberg, Berlin, pp 487-556

19. Wernicke C (1874) Der aphasische Symptomenkomplex. Eine psychologische Studie auf Anatomischer Basis. Cohn und Weigert, Breslau

20. Willmes K, Poeck K (1993) To what extent can aphasic syndrome be localized? Brain 116:15271540 